\title{
Refining surgical therapy of liver cancer in cirrhosis: etiology makes the difference
}

\author{
Massimo Colombo ${ }^{1}$, Ana Lleo ${ }^{1,2}$ \\ ${ }^{1}$ Humanitas Clinical and Research Center, Rozzano, Italy; ${ }^{2}$ Department of Biomedical Sciences, Humanitas University, Pieve Emanuele, Italy \\ Correspondence to: Massimo Colombo, MD. Head, Center for Translational Research in Hepatology. Humanitas Clinical and Research Center, Via A. \\ Manzoni 56, 20089 Rozzano, Italy. Email: mcolombo46@yahoo.it; massimo.colombo@humanitas.it. \\ Provenance: This is an invited Editorial commissioned by Section Editor Guwei Ji (Liver Transplantation Center, The First Affiliated Hospital of \\ Nanjing Medical University, Nanjing, China). \\ Comment on: Pinna AD, Yang T, Mazzaferro V, et al. Liver Transplantation and Hepatic Resection can Achieve Cure for Hepatocellular Carcinoma. \\ Ann Surg 2018;268:868-75.
}

Received: 10 November 2018; Accepted: 28 November 2018; Published: 14 December 2018.

doi: $10.21037 / \operatorname{tgh} .2018 .12 .03$

View this article at: http://dx.doi.org/10.21037/tgh.2018.12.03

As treatment of hepatocellular carcinoma (HCC) has evolved from an empirical approach to remove the visible tumour towards an evidence-based decision making algorithm, liver transplantation (LT) has progressively emerged as the only curative option for accurately selected patients with a small tumor restricted to the liver (1). Indeed, compared to non-transplant radical therapies like hepatic resection (HR) and local ablation, LT has the added value of preventing complications related to portal hypertension caused by the underlying liver disease and removing the source of recurrences, thereby representing the best option in patients with deteriorated liver function (2-4). Working against this strategy, however, are scarce organ donations worldwide that limit patient access to LT, a fact that has led to the application of strict rules for listing and has fueled the debate on to what extent HR and local ablation therapies can compete with LT in real life practice treatment of patients with a limited HCC burden. With respect to HR, the argument has further been amplified by the lack of a systematic comparison of the impact of therapy on the residual entire life-expectancy of patients, the so-called statistical cure, therefore making difficult to appreciate the full benefits of an option over the other especially in the horizon of cost effectiveness.

In an attempt to contribute to this debate, Pinna and colleagues retrospectively scrutinized a large cohort of surgically treated patients collected between 2000 and 2016 in 7 internationally reputed surgical units located in both hemispheres. The chance of obtaining a statistical cure of HCC, which measures when the mortality of patients treated for a specific disease returns to the value expected in the general population, was evaluated on an intentionto-treat (ITT) basis in 1,218 patients undergoing LT and 2,068 treated with HR who were stratified by preoperative clinical and tumor features. In particular, liver transplanted patients considered in this survey had no macrovascular invasion by tumor cells nor incidentally detected tumors, whereas listing to LT was possible also for patients with previous treatments of HCC and with a tumor burden beyond Milan criteria. All in all, criteria of HR were looser than those recommended by the European Association for the Study of the Liver (5) as they included patients with clinically significant portal hypertension without any stratification for presence of cirrhosis, uptake of antiviral therapy and biologically aggressiveness of the tumor as defined by serum levels of alfa fetoprotein. Interestingly enough, rates of successful down staging were rather low (27\%) and accompanied by short waiting times (2.3 months), a condition known to contribute increasing the risk of tumor recurrence (6). This notwithstanding, the cure fraction intended as disease-free survival was strikingly higher following LT compared to HR (74.1\% vs. $24.1 \%$ ), making LT to outperform HR within all transplant criteria considered, especially patients with multiple tumors and even in presence of a drop-out up to $20 \%$. However, the effect size of LT over HR in terms of a 
cure dropped significantly in the set of patients with oligo nodular tumors, became small for a drop-out up to $20 \%$ and negligible for single tumors below $5 \mathrm{~cm}$ in size. These findings led the authors to conclude that, on the basis of the higher chances of providing longer tumor-free survival, LT outperforms HR, whereas in presence of restricted listing and long waiting-times with a high risk of drop-out, patients with a small tumor treated with HR may gain as many cure probabilities as with a LT. Instead, LT stands as the preferable choice in patients with multiple HCC nodules.

While we acknowledge that the message delivered by this study might help refining the therapeutic algorithm of liver cancer in selected subgroups of patients, conclusions of the authors should cautiously be taken before external validation of their findings become available. In fact, it should not be overlooked that the power of this study is challenged by substantial referral biases reflecting recruitment of heterogeneous populations of HCC patients from units located in various geographical areas that are guided by recommendations crafted by different professional society (7). On top of this, many hepatologists would be reluctant to accept the working hypothesis of the study that tumour recurrence after HR is treatable to the point of not interfering with mortality rates, a founding assumption that conflicts with robust data in literature identifying recurrence as the leading cause of late onset mortality after resection of a small tumor in cirrhosis (5). While the study by Pinna and colleagues has the undisputed merit of matching HR and LT outcomes in terms of ITT and to expand the time horizon of follow-up, let me remind that decades ago in Barcelona an ITT analysis identified the length of waiting time and severity of portal hypertension as drivers of the choice between HR and LT in patients with cirrhosis and a small HCC (6).

As a clinical cure of viral hepatitis $B$ and $C$ is now at hand, we look forward to having a validation study of the statistical cure done in HCC patients with pharmacologically inactivated viral hepatitis as it might substantially contribute

doi: $10.21037 / \operatorname{tgh} .2018 .12 .03$

Cite this article as: Colombo M, Lleo A. Refining surgical therapy of liver cancer in cirrhosis: etiology makes the difference. Transl Gastroenterol Hepatol 2018;3:104. to further refining criteria of surgical therapy of HCC and even subverting therapeutic algorithms consolidated by ages.

\section{Acknowledgements}

None.

\section{Footnote}

Conflicts of Interest: The authors have no conflicts of interest to declare.

\section{References}

1. Forner A, Reig M, Bruix J. Hepatocellular carcinoma. Lancet 2018;391:1301-14.

2. Poon RT, Fan ST, Lo CM, et al. Difference in tumor invasiveness in cirrhotic patients with hepatocellular carcinoma fulfilling the Milan criteria treated by resection and transplantation: impact on long-term survival. Ann Surg 2007;245:51-8.

3. Koniaris LG, Levi DM, Pedroso FE, et al. Is surgical resection superior to transplantation in the treatment of hepatocellular carcinoma? Ann Surg 2011;254:527-37; discussion 537-8.

4. Mazzaferro V, Chun YS, Poon RT, et al. Liver transplantation for hepatocellular carcinoma. Ann Surg Oncol 2008;15:1001-7.

5. European Association for the Study of the Liver. EASL Clinical Practice Guidelines: Management of hepatocellular carcinoma. J Hepatol 2018;69:182-236.

6. Llovet JM, Fuster J, Bruix J. Intention-to-treat analysis of surgical treatment for early hepatocellular carcinoma: resection versus transplantation. Hepatology 1999;30:1434-40.

7. Omata M, Cheng AL, Kokudo N, et al. Asia-Pacific clinical practice guidelines on the management of hepatocellular carcinoma: a 2017 update. Hepatol Int 2017;11:317-70. 